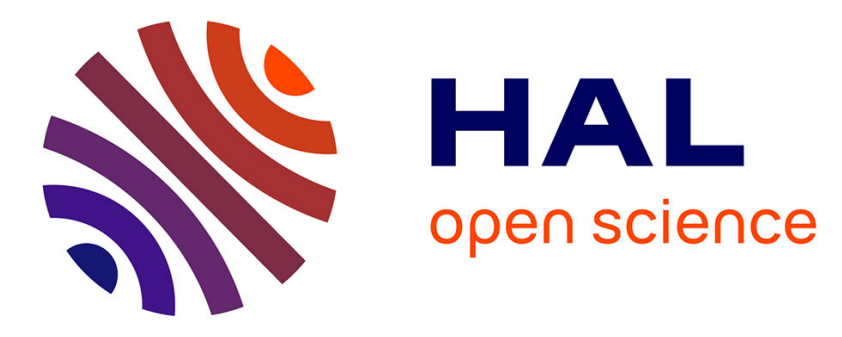

\title{
Silicon Photonics Based on Ge/SiGe Quantum Well Structures
}

D M Marris-Morini, Vladyslav Vakarin, Papichaya Chaisakul, Jacopo Frigerio,

Mumtahina Rahman, Joan-Manel Ramírez, Mohamed-Said Rouifed, Daniel

Chrastina, Xavier Le Roux, Giovanni Isella, et al.

\section{To cite this version:}

D M Marris-Morini, Vladyslav Vakarin, Papichaya Chaisakul, Jacopo Frigerio, Mumtahina Rahman, et al.. Silicon Photonics Based on Ge/SiGe Quantum Well Structures. 18th International Conference on transparent Optical Networks (ICTON 2016), Jul 2016, Trente, Italy. 10.1109/ICTON.2016.7550395 . hal-01388130

\section{HAL Id: hal-01388130 \\ https://hal.science/hal-01388130}

Submitted on 26 Oct 2016

HAL is a multi-disciplinary open access archive for the deposit and dissemination of scientific research documents, whether they are published or not. The documents may come from teaching and research institutions in France or abroad, or from public or private research centers.
L'archive ouverte pluridisciplinaire HAL, est destinée au dépôt et à la diffusion de documents scientifiques de niveau recherche, publiés ou non, émanant des établissements d'enseignement et de recherche français ou étrangers, des laboratoires publics ou privés. 


\title{
Silicon Photonics Based on Ge/SiGe Quantum Well Structures
}

\author{
D. Marris-Morini ${ }^{1}$, V. Vakarin $^{1}$, P. Chaisakul ${ }^{1,3}$, J. Frigerio ${ }^{2}$, M. Rahman ${ }^{1}$, J. M. Ramírez ${ }^{1}$, M-S. Rouifed ${ }^{1}$, \\ D. Chrastina ${ }^{2}$, X. Le Roux ${ }^{1}$, G. Isella ${ }^{2}$, L. Vivien ${ }^{1}$ \\ 1 Institut d'Electronique Fondamentale, Univ. Paris-Sud, CNRS UMR 8622, Orsay, France \\ 2 L-NESS, Politecnico di Milano, Polo di Como, Italy \\ 3 Department of Materials Engineering, The University of Tokyo, Japan \\ delphine.morini@u-psud.fr
}

\begin{abstract}
Recent advances in the theoretical and experimental studies of $\mathrm{Ge} / \mathrm{SiGe}$ quantum wells structures will be presented with a focus on optical telecommunication applications. Firstly, high-speed stand-alone $\mathrm{Ge} / \mathrm{SiGe}$ QW electro-absorption modulators and photodetectors will be reported. It will be followed by the presentation of different methods for engineering Ge/SiGe QW to tune the operating wavelength to $1.3 \mu \mathrm{m}$. Then the demonstration of a strong electro-refraction Ge/SiGe QW will be reported. This effect could be used to achieve optical modulation but requires the embedding of the QWs in a Mach-Zehnder interferometer. Finally it will be shown that these active devices can be combined with advanced passive structures using Ge-rich SiGe virtual substrate on graded buffer as a waveguide.
\end{abstract}

\section{Introduction}

The interest in silicon photonics is continuously growing as it is now considered to have potential applications in telecommunication and data communications. In this context, Ge has been proposed by a few research groups as the material of choice for the realization of a complete photonic circuit. Despite being an indirect band gap material, its absorption is "direct gap like" thanks to the small energy difference between the direct and indirect bandgaps. Moreover, it is a group IV material, compatible with fabrication in CMOS foundries. Among Ge-based structures, Ge rich-Ge/SiGe quantum wells $(\mathrm{QW})$ have received a growing interest, as the quantum confinement in Ge wells allow additional features with respect to the bulk materials, such as more abrupt band-edge absorption, the presence of excitonic features as well as the possibility of band structure engineering.

The first demonstration of a direct gap-related optical property in Ge/SiGe QW was the observation of the quantum-confined Stark effect (QCSE) [1,2]. These results paved the way to a number of exciting works addressing not only the electro-absorption but also the electro-refraction [3] mechanisms in Ge/SiGe QW structures and tackling the realization of innovative optoelectronic devices.

Recent advances in the theoretical and experimental studies of $\mathrm{Ge} / \mathrm{SiGe}$ quantum wells structures will be presented with a focus on optical telecommunication applications. Firstly, high-speed stand-alone $\mathrm{Ge} / \mathrm{SiGe} \mathrm{QW}$ electro-absorption modulators and photodetectors will be reported. It will be followed by the presentation of different methods for engineering Ge/SiGe QW to tune the operating wavelength to $1.3 \mu \mathrm{m}$. Finally it will be shown that these active devices can be combined with advanced passive structures using Ge-rich SiGe virtual substrate on graded buffer as a waveguide.

\section{High speed modulator and photodetector}

QCSE leads to a strong variation of absorption coefficient around the absorption band-edge of $\mathrm{Ge} / \mathrm{SiGe} \mathrm{QW}$ structures. This effect has first been used to demonstrate high speed modulation. The stand-alone modulator is reported in Fig 1. It is based on a $3 \mu \mathrm{m}$-wide, $90 \mu \mathrm{m}$-long waveguide made of $\mathrm{Ge} / \mathrm{Si}_{0.15} \mathrm{Ge}_{0.85} \mathrm{QW}$ inserted in a PIN diode. The $\mathrm{QW}$ are grown on a $\mathrm{Si}_{0.1} \mathrm{Ge}_{0.9}$ relaxed buffer using a strain compensated design, and a $13 \mu \mathrm{m}$-thick graded buffer between $\mathrm{Si}$ and $\mathrm{Si}_{0.1} \mathrm{Ge}_{0.9}$ is used to manage the lattice mismatch between $\mathrm{Si}$ and $\mathrm{Si}_{0.1} \mathrm{Ge}_{0.9}$ and to minimize the threading dislocations density [4]. 

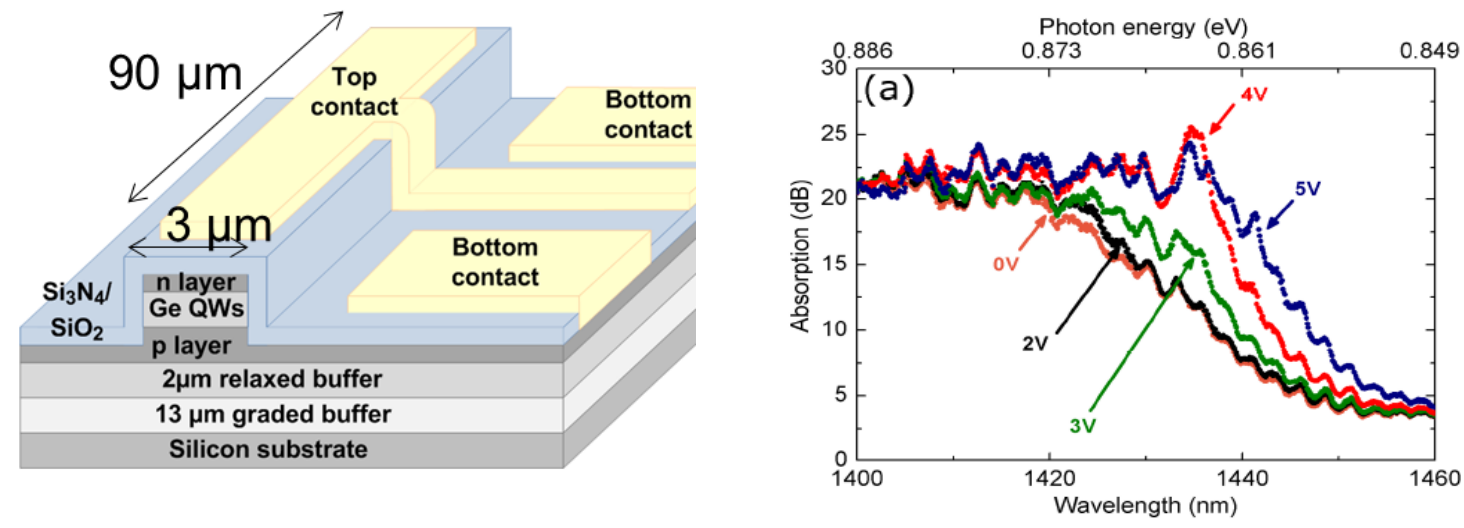

Fig. 1. (a) Schematic view of a Ge/SiGe QW structure in a waveguide configuration

that can be used as a modulator or as photodetector. (b) absorption spectra as a function of the applied voltage on the modulator.

A high extinction ratio up to more than $10 \mathrm{~dB}$ is obtained while an extinction ratio larger than $6 \mathrm{~dB}$ is reported in a $20 \mathrm{~nm}$ wavelength range (Fig 1b). The electro-optic bandwidth is measured larger than $20 \mathrm{GHz}$, while the power consumption is less than $100 \mathrm{fJ} / \mathrm{bit}$. Interestingly, as the modulator is based on electro-absorption, the same active region can be used for photodetection. Responsivity between 0.5 and $0.8 \mathrm{~A} / \mathrm{W}$ is obtained in a $80 \mu \mathrm{m}$-long waveguide. $10 \mathrm{Gbit} / \mathrm{s}$ operation was demonstrated [5].

\section{Ge/SiGe quantum well engineering}

As a main advantage, QW can be engineered to obtain significant QCSE around the telecommunication wavelength of $1.3 \mu \mathrm{m}$. In the first demonstrations, $\mathrm{Ge} / \mathrm{Si}_{0.15} \mathrm{Ge}_{0.85} \mathrm{QW}$ grown on $\mathrm{Si}_{0.1} \mathrm{Ge}_{0.9}$ virtual substrate lead to QCSE at 1.42 $\mu \mathrm{m}$. Different methods can be used to decrease the operating wavelength. Firstly, knowing that a higher level of compressive strain on Ge allows increasing its bandgap energy, a design has been proposed based on $\mathrm{Ge} / \mathrm{Si}_{0.35} \mathrm{Ge}_{0.65}$ QW grown on $\mathrm{Si}_{0.21} \mathrm{Ge}_{0.79}$. As predicted, a strong absorption variation has been obtained at $1.3 \mu \mathrm{m}$ [6] (Fig 2a). A second way to decrease the operating wavelength of the widely-used $\mathrm{Ge} / \mathrm{Si}_{0.15} \mathrm{Ge}_{0.85} \mathrm{QW}$ grown on $\mathrm{Si}_{0.1} \mathrm{Ge}_{0.9}$ is to increase QW confinement energy by varying the quantum well thickness [7]. As an example, the absorption spectra of a structure made of $6.5 \mathrm{~nm}$-thick Ge well is reported in Fig. 2b, showing a strong QCSE within the O-band telecommunication wavelength, verifying the potential to use this structure for high extinction ratio and moderate absorption loss modulator over 30-nm-wide spectral range from 1330 to $1360 \mathrm{~nm}$.
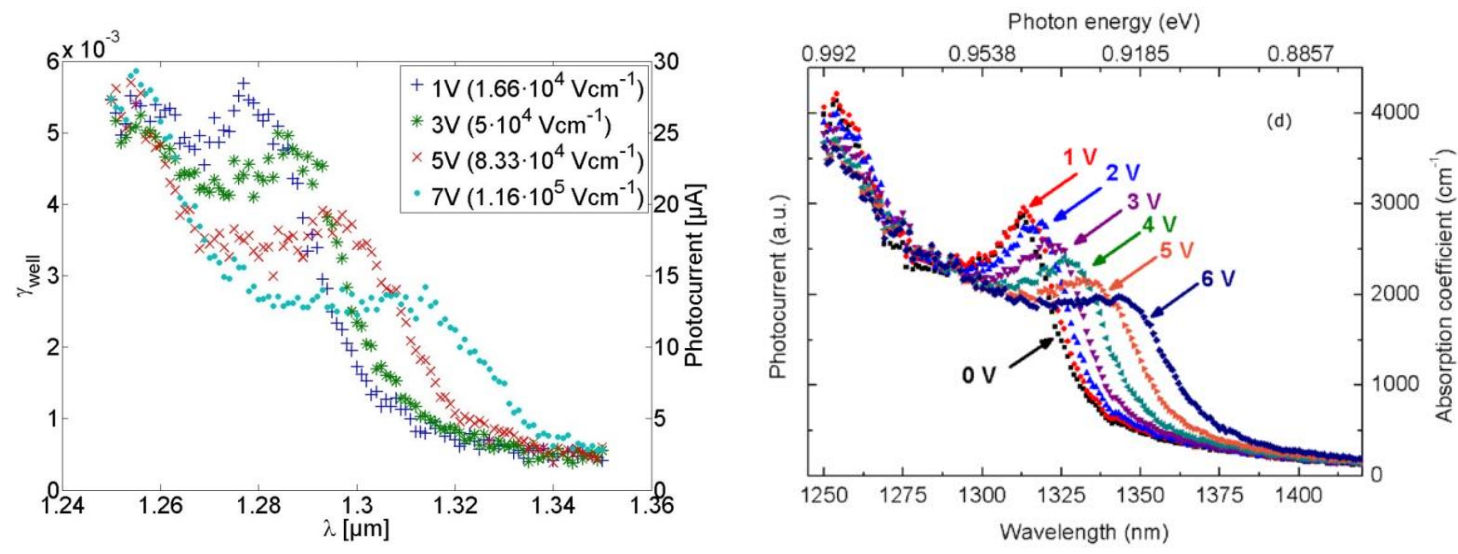

Fig. 2. QCSE in the O-band telecommunication wavelength, based on (a) strain envineering, (b) thickness engineering of the QW structures 


\section{Combination with advanced passive structures}

The main challenging point of $\mathrm{Ge} / \mathrm{SiGe} \mathrm{QW}$-based devices is their integration with silicon on insulator (SOI) waveguides because of the need of a virtual substrate between $\mathrm{Si}$ and Ge-rich structures. We proposed to integrate $\mathrm{Ge} / \mathrm{SiGe} \mathrm{QW}$ devices with SiGe waveguides using the linear index increasing in the graded buffer. Light can then be coupled in the QW using tapered waveguides for the active devices as illustrated in Fig $3 \mathrm{a}$. As a main challenge the Ge concentration in the SiGe waveguide should allow simultaneously a low loss waveguide and a good crystalline quality of the $\mathrm{Ge} / \mathrm{SiGe} \mathrm{QW}$ active region. Using this approach, an optical link based on a passive $\mathrm{Si}_{0.16} \mathrm{Ge}_{0.84}$ waveguide and optoelectronic $\mathrm{Ge} / \mathrm{Si}_{0.16} \mathrm{Ge}_{0.84}$ QWs devices including an optical modulator and a photodetector has been demonstrated. [8] (Fig 3b)
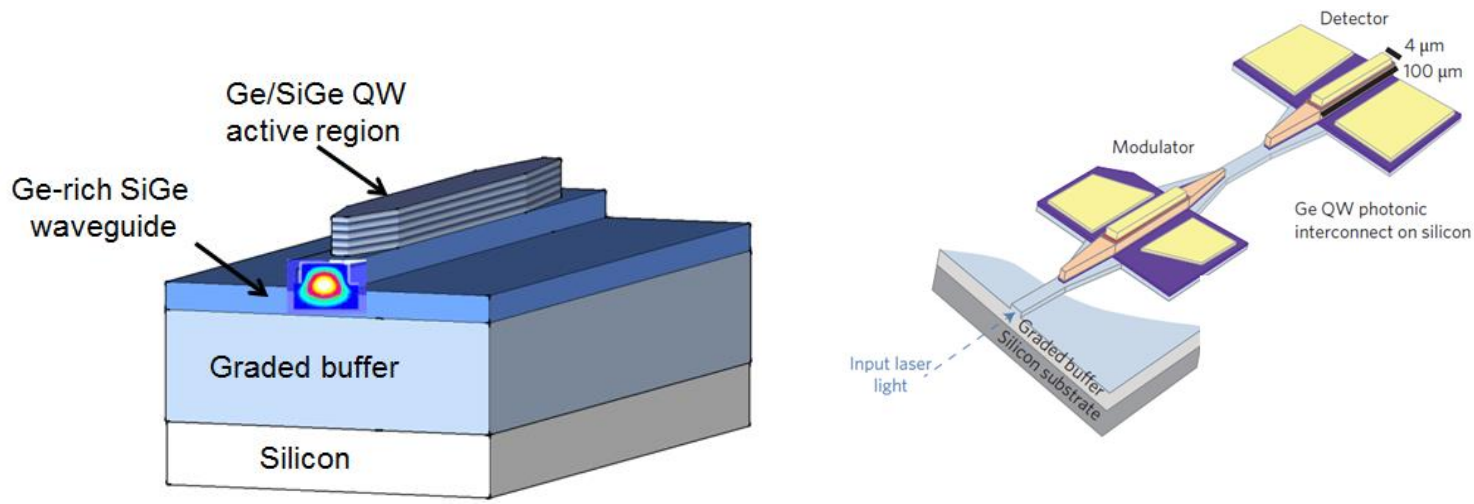

Fig. 3. Integration of Ge/SiGe QW devices with Ge-rich SiGe waveguide on graded SiGe buffer. (a) SiGe waveguides coupled with the QW active region ; (b) : optical link based on a modulator, a waveguide and a photodetector

In addition we recently demonstrated the possibility to use this new Ge-rich SiGe on graded buffer waveguide as a competitive platform for photonic integration: sharp bends and an integrated Mach-Zehnder interferometer including SiGe waveguides and a relatively compact beam splitter were demonstrated from Ge-rich SiGe waveguide on graded buffer on silicon substrate [9], based on optical confinement tuning between slightly etched and deeply etched waveguides. These results pave the way toward the monolithic integration of Ge-based active devices with efficient and compact passive Ge-rich SiGe optical circuitry on bulk silicon wafers.

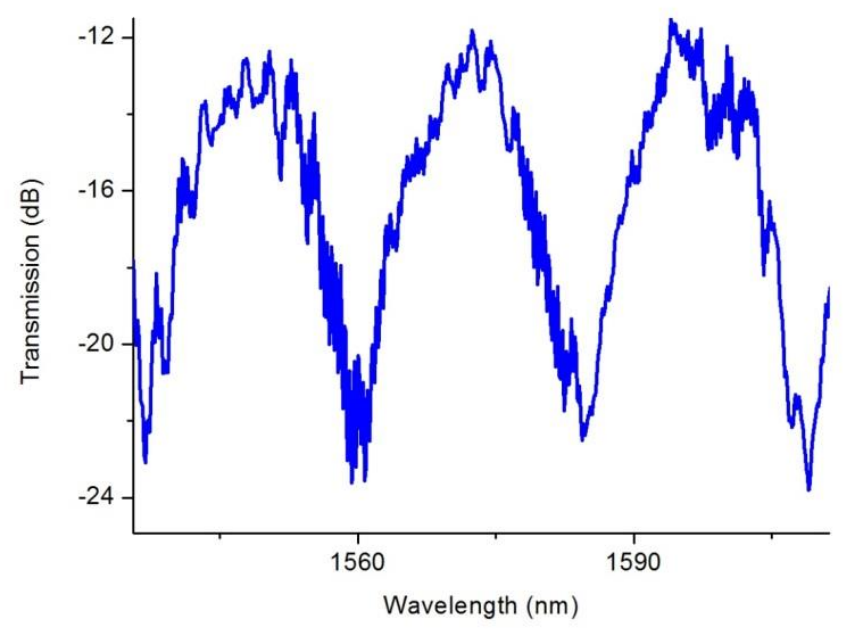

Fig. 4. Optical transmission of the asymmetric Mach-Zehnder interferometer made of Ge-rich SiGe waveguide on graded SiGe buffer 


\section{Acknowledment}

This work is supported by the CARIPLO foundation through the grant EIDOS 2011-038, by Marie Curie International Outgoing Fellowships through grant agreement PIOF-GA-2013-629292 and by the European Research Council under the ERC Starting Grant INsPIRE nº639107.

\section{References}

[1] Y. Kuo et al., Nature, 437, 1334 (2005).

[2] P. Chaisakul et al, Optics Letters, 35 (17) 2913 (2010).

[3] J. Frigerio et al., Applied Physics Letters 102, 061102 (2013).

[4] P. Chaisakul et al., Optics Express, 20 (3), 3219-3225, (2012)

[5] P. Chaisakul et al., IEEE Photonics Technology Letters, 23 (20), (2011).

[6] M-S. Rouifed. et al., Optics Letters 37, 3960 (2012).

[7] P. Chaisakul et al., Journal of Applied Physics, 116, 193103 (2014).

[8] P. Chaisakul et al., Nature Photonics 8, 482-488 (2014).

[9] V. Vakarin et al., Optics Express, 23 (24) 30821 (2015). 